\title{
UNA MIRADA A LA VALORACIÓN EMPRESARIAL COMO MÉTODO AUTORITARIO EN LAS MIPYMES
}

Autores: Carlos Pacheco Ruiz (1) Jorge Bermúdez Fuentes (2)

\section{RESUMEN}

7 ste estudio tiene como propósito mostrar la valoración de empresas como forma autoritaria para la comprensión de $\triangle$ valor en las Mipymes. Basados en la revisión oportunista de la literatura económica y financiera, se realizó un ejercicio de reflexión apoyado en los distintos modelos de valoración empresarial aplicables a las Mipymes, que llevaron a mostrar algunas concepciones de valor y medición, difundidas en el mundo y que son vistas como fiables, teniendo como factor clave para determinar un método de valoración, el saber decidir qué tan avanzado es el modelo de negocio. Se concluye que para la toma de decisiones, los propietarios usan modelos documentados o mentales basados en el multicriterio, los métodos de valoración de empresas son dinámicos y se adaptan a las transformaciones sociales y empresariales brindando seguridad y una supuesta precisión que es aceptada bajo mediciones por autoridad, teniendo en cuenta que el valor se basa en estimaciones y juicios en lugar de verdades absolutas. .

Palabras claves: Valor, métodos de valoración, objetividad, autoridad

\section{ABSTRACT}

T he purpose of this study is to show the valuation of companies as an authoritarian way to understand value in MSMEs. Based on the opportunistic review of the economic and financial literature, a reflection exercise was carried out based on the different models of business valuation applicable to MSMEs, which led to show some conceptions of value and measurement, disseminated throughout the world that are seen as reliable, having as a key factor to determine a valuation method, knowing how to decide how advanced is the business model. It is concluded that for decision making, the owners use documented or mental models based on the multicriteria, the business valuation methods are dynamic and adapt to the social and business transformations providing security and a supposed accuracy that is accepted under measurements by authority, taking into account that the value is based on estimates and judgments instead of absolute truths .

Keywords: Value, valuation methods, objectivity, authority.

\footnotetext{
El presente artículo está basado en la conferencia: Medición y valoración contable, una aproximación heterodoxa, presentada por el profesor Mauricio Gómez Villegas de la Universidad Nacional en el tercer Simposio Internacional de Contametría.

(1) Administrador de empresas y docente universitario en Universidad de Sucre. Especialista en Gerencia de Proyectos. Magister en Administración. (2) Estudiante de administración de empresas, universidad de Sucre
} 


\section{UNA MIRADA A LA VALORACIÓN EMPRESARIAL COMO MÉTODO AUTORITARIO EN LAS MIPYMES}

\section{INTRODUCCIÓN}

$\mathrm{L}$ as Micro, pequeñas y medianas empresas (Mipyme) son de importancia fundamental para el crecimiento y la creación de empleo en una economía Behr (2013). Estas aparecen como motor de crecimiento y fuente de empleo en países de envergadura económica como Japón o Alemania, por citar sólo algunos ejemplos (Uccelatore, 2012). Según Saavedra \& Hernandez (2008) las Mipymes generan entre el $44 \%$ y $70 \%$ del empleo total en países desarrollados, en los países en vía de desarrollo entre el $50 \%$ y $80 \%$ del empleo y en los países industrializados, por regla general, las Mipymes representan más del 90\% de las empresas (Baas T. e M. Schroorten, 2006). En la Unión Europea, en particular, la promoción de las Mipyme se considera vital para aumentar su competitividad (Van Praag C. M. e P. H. Versloot, 2007), ratificando así que este segmento representan un rol trascendental de carácter social y económico.

En Colombia el sector empresarial se clasifica en micro, pequeñas, medianas y grandes empresas y está reglamentado en Ley 590 de 2000, modificada por las Leyes 905 de 2004, 1111 de 2006, 1151 de 2007 y 1450 de 2011. En el país hay una marcada presencia de las Mipymes, teniendo en cuenta que el $80.8 \%$ del empleo generado en Colombia proviene de ellas, proporcionalmente las micro ofrecen el $50.3 \%$ y las pequeñas y medianas hacen el otro $30.5 \%$ de generación de puestos de trabajo (Revista Dinero, 2016).

Con respecto a su valoración, los economistas han hechos ingentes esfuerzos por explicar su naturaleza en un maremágnum de relaciones que permiten mirar su significado desde diferentes perspectivas, lo que hace indispensable una aproximación a las relaciones intrínsecas y al conocimiento que dibuja la economía y la administración financiera de negocios en torno al valor de las empresas.
Los modelos de valor intentan explicar la realidad basados en una cosmovisión compartida y adoptada, especialmente por los financistas, y que en el buen sentido, irrumpen en la vida organizacional casi de manera autoritaria; se piensa en lo que constituye el costo, en una cascada transaccional que lleva cada vez a más valoraciones sin detenerse a reflexionar en la forma de pensamiento que guía la resolución de esos conflictos que muchas veces se creen resueltos, apoyados en consensos y sobre los hombros de una masa que no cuestiona qué es lo que realmente significa el valor, no discute si lo que supone que es una medida fiel de la realidad, tal vez no lo es tanto, pero sí resulta a la medida de las necesidades de explicación de las operaciones y manejo de riqueza de las organizaciones.

Se plantea el mostrar no solo las formas de valoración tradicionales sino una percepción reflexiva del valor, la objetividad y la autoridad de las ciencias sociales, teniendo en cuenta que las finanzas como rama de la economía, estudia las formas en que los individuos, entidades empresariales y otras organizaciones asignan recursos a través del tiempo y toman decisiones en presencia de incertidumbre y riesgo (Universidad EAFIT, 2017) o bajo condiciones de "escasez" o de "abundancia", por lo que las técnicas de valoración se convierten en guías con un tinte de autoridad, puesto que las ciencias sociales tienden a mostrarle al hombre su realidad y a explicar cómo interactuamos, en este caso alrededor del concepto de valor. 


\section{UNA MIRADA A LA VALORACIÓN EMPRESARIAL COMO MÉTODO AUTORITARIO EN LAS MIPYMES}

\section{DESARROLLO}

$\int$ as pymes afrontan múltiples problemas y estimar su valor dentro del cuadro de posibilidades que ofrece el ejercicio depende en gran medida de las perspectivas futuras de ganancias y de darse un cambio en el cuadro de mando, la toma de decisiones determina, como es normal, tales perspectivas. Una valoración adecuada parte de la objetividad con que se mire a la unidad empresarial, en la que es muy importante observar la solidez de los activos. Al evaluar a las Mipymes, se deben tomar en cuenta sus particularidades, como el hecho de no ser fuertes en materia de planeación y control de procesos, que traen como consecuencia mayores dificultades a la hora de anticiparse a los cambios del entorno, situación que puede entrar a condicionar la elección del método de valoración. Además, el propietario se enfrenta al problema de ser objetivo y pensar en la objetividad que cada modelo le brinda, estando sujeto a formas que resultan autoritarias en cuanto a saber qué tanto representa su empresa en términos de valor.

La expresión objetividad es utilizada corrientemente por la mayoría de las personas cuando desean expresar que al evaluar una situación, se atienen exclusivamente a los hechos. El diccionario de la lengua española, por su parte, define la calificación objetivo por su aplicación a lo que existe realmente, fuera del sujeto que lo conoce. Ambas perspectivas son insostenibles desde el punto de vista de la epistemología subyacente en cualquiera de las múltiples propuestas teóricas de la psicología, por cuanto suponen que el observador puede prescindir a voluntad de su propia subjetividad (Morales de Barbenza, 2001)

Recordando al poeta Ramón de Campoamor

Nada es verdad ni mentira; todo es según el color del cristal con que se mira.
Pretender llegar a una observación objetiva de un sistema de complejidad y variedad infinitas, supone previa y necesariamente reducir drásticamente su complejidad y su variedad, hasta convertirla en finita, limitado y totalmente descriptible (reduccionismo) (Henric Coll, 2009).

Puesto que el valor responde al sentido humano creado para tal concepto, es bueno anotar que para el objetivismo tradicional el valor depende por completo del objeto y existe independientemente de la voluntad y la conciencia valorativa del sujeto. Para las concepciones subjetivistas el valor depende por completo de la subjetividad, individual o colectiva, independientemente de las características del objeto. La debilidad de estas concepciones estriba en que la subjetividad individual o colectiva puede estar errada (Sánchez Hernández, 2005).

Entonces, si la teoría subjetiva del valor considera el valor de un objeto como algo no inherente, resultaría que un mismo objeto vale más para diferentes personas en función de cuánto lo desean o necesitan. Por otro lado, se podría crear o aumentar el valor de un objeto transfiriendo la propiedad del mismo a un ente que lo mantenga con un valor superior, sin que haya la necesidad de modificarlo, teniendo en cuenta que existen elementos como la cultura, los sensaciones y la disponibilidad, que pueden llegar a influir en el valor.

Haciendo un traslado a la objetividad social, se puede decir que es, en alguna medida, subjetividad objetivada, es decir, producto de la actividad humana, pero que trasciende, por su significación, a la propia subjetividad que la crea y se inserta con un lugar propio en el sistema de relaciones sociales (Fabelo Corzo, 2004) 


\section{UNA MIRADA A LA VALORACIÓN EMPRESARIAL COMO MÉTODO AUTORITARIO EN LAS MIPYMES}

En tal sentido, tal vez no existe el valor absoluto, incluso el dinero solo vale lo que las convenciones dictan que vale, convirtiéndose simplemente en un medio de intercambio aceptado socialmente. Aquí entra algo muy importante, y es la autoridad, que se legitima por el reconocimiento intersubjetivo de todos los sujetos que colaboran en un ámbito funcional, referente a la capacidad moral para dirigir una función, dentro de la que se inserta la racionalidad de las acciones que son validadas por una colectividad, debido a que se reconoce que alguien más es superior en juicio y que es capaz de percibir algo mejor que uno mismo (Aristóteles, 2000 y Gadamer, 2000).

Legitimar la autoridad proyecta mecanismos de articulación de todos los actores, dentro de un ámbito de reconocimiento intersubjetivo y al margen del carácter impositivo que genera el poder; en sí misma, la figura de la autoridad conjunta el esfuerzo colectivo mediante la coordinación de todos sus componentes (Cruz Soto, 2010)

Si la sociedad es una masa de acuerdos y las ciencias sociales nos explican cómo se comporta, cómo se relacionan los individuos, cómo se consolida la acción social, entonces se tiene un vínculo autoritario sobre cómo se hacen las cosas en sociedad. Ahora, entendiendo dentro de las ciencias sociales a la economía y a las finanzas como parte de esta, los modelos de valoración (en este caso financieros) también son una construcción social de tinte autoritario. Es como creer que así debe ser, entrando a aplicar, más que a cuestionar.

Ligado a lo anterior, está la forma en que se hacen las mediciones. Al respecto la UNAM (2017), rescata conceptos como el de Bachelard (1972), quien sugirió que hay que reflexionar para medir, y no medir para reflexionar. En ese mismo escrito se destaca que Torgerson (1967) señala tres diferentes clases de medición:

\section{Medición Fundamental.}

La primera, la medición fundamental, depende de las leyes que relacionan a las cantidades entre sí, que representan a una construcción o sistema determinado. Los números se asignan siguiendo las leyes naturales y no se presupone la medición de otras variables.

\section{Medición Derivada.}

Con respecto a la medición derivada, Torgerson señala que las propiedades numéricas, adquieren significado a través de leyes que relacionan, no a las cantidades de una construcción, como en el caso de la medición fundamental, sino a las propiedades entre sí.

\section{Medición por Autoridad.}

En esta clase de medición, las propiedades medidas adquieren significado a través de un procedimiento un tanto arbitrario que se basa, en gran medida, en la intuición. Se asume que existe una relación entre las observaciones realizadas y el concepto o construcción de interés. Ejemplo de esta clase de medición se tiene en los índices que se utilizan en Psicología, Sociología y Economía. Empero, no deberá perderse de vista que el mayor problema de la medición por autoridad, reside en que una construcción o concepto determinado puede ser definido de muy diversas maneras, por la carencia de sistemas teóricos previamente establecidos, evidenciando con esto, la necesidad de dedicar un mayor esfuerzo al logro de mediciones fundamentales para eliminar mucha de la arbitrariedad que actualmente se encuentra presente en el proceso de medición que siguen las ciencias sociales y las de la conducta. 


\section{UNA MIRADA A LA VALORACIÓN EMPRESARIAL COMO MÉTODO AUTORITARIO EN LAS MIPYMES}

Esta última forma de medición es la que más se compenetra con las ideas que se muestran en el presente escrito, al ser el tipo de medición propio de las ciencias sociales en donde se miden interacciones sociales sobre las que no existen objetos materiales sino relaciones fluctuantes de los sujetos (Gómez Villegas, 2016) y deriva en las formas consideradas adecuadas para enfrentar el proceso de valoración empresarial.

La valoración de una empresa se puede definir como el proceso mediante el cual se busca la cuantificación de los elementos que constituyen su patrimonio, su actividad, su potencialidad o cualquier otra característica de la misma susceptible de ser valorada (Pérez-Cotapos \& Silva Palaveci, 2009).

Hay una diferencia existente entre el valor de una empresa y su precio, el valor se define como el beneficio obtenido por la adquisición de esta, este es subjetivo y depende de quien la esté evaluando es por esto que al determinar el valor lo que se obtiene es un intervalo dentro del cual se encuentra el valor definitivo y por otro lado el precio es lo que se paga por la adquisición de dicha empresa el cual queda determinado por mutuo acuerdo de las partes (Millanao M. \& Saavedra R., 2011).

\section{Métodos de Valoración:}

Fuente: Millanao M. \& Saavedra

\begin{tabular}{|c|c|c|c|c|c|}
\hline BALANCE & $\begin{array}{l}\text { CUENTADE } \\
\text { RESULTADOS }\end{array}$ & $\begin{array}{c}\text { MXXTOS } \\
\text { (GOODWLL) }\end{array}$ & $\begin{array}{l}\text { DESCUENTOO } \\
\text { FLUNOS }\end{array}$ & $\begin{array}{c}\text { CREACION DE } \\
\text { VALOR }\end{array}$ & OPCIONES \\
\hline Valor contable & Múltiplos & Clásico & Free cash flow & EVA & Blacky Scholes \\
\hline $\begin{array}{l}\text { Valor contable } \\
\text { ajustado }\end{array}$ & Beneficio: PER & $\begin{array}{l}\text { Unión de } \\
\text { expertos }\end{array}$ & $\begin{array}{l}\text { Cash flow } \\
\text { acciones }\end{array}$ & $\begin{array}{l}\text { Beneficicio } \\
\text { económico }\end{array}$ & $\begin{array}{l}\text { Opción de } \\
\text { invertir }\end{array}$ \\
\hline Valor de liquidación & Ventas & $\begin{array}{l}\text { Contables } \\
\text { europeos }\end{array}$ & Dividendos & $\begin{array}{l}\text { Cash value } \\
\text { added }\end{array}$ & $\begin{array}{l}\text { Ampliar el } \\
\text { proyecto }\end{array}$ \\
\hline Valor sustancial & Ebitda & $\begin{array}{c}\text { Renta } \\
\text { abreviada }\end{array}$ & $\begin{array}{l}\text { Capital cash } \\
\text { flow }\end{array}$ & CFROI & $\begin{array}{l}\text { Aplazar la } \\
\text { inversión }\end{array}$ \\
\hline Activo neto real & Otros mútiplos & Otros & APV & & $\begin{array}{l}\text { Usos } \\
\text { alternativos }\end{array}$ \\
\hline
\end{tabular}

En este sentido, la literatura existente muestra estos métodos de valoración de las empresas a nivel internacional, dividiéndolos en enfoque de ingresos, enfoque de mercado y enfoque de activos. Para pre- sentarlos, se utiliza la distribución de Damodaran (2005) y Fernández (2013):

1. Discounted cash flow (DCF) valuation

2. Liquidation and accounting valuation

3. Relative valuation

4. Contingent claim valuation (real options)

5. Goodwill valuation

Por otra parte Aznar Bellver, Cayo Araya, \& Cevallos Varela (2016), dicen que la empresa no es un ente aislado que funciona independientemente del entorno que le rodea, por eso al abordar la valoración de una empresa hay que tener en cuenta multitud de factores tanto internos como externos, en principio todos aquellos que pueden afectar a su valor bien sea positiva o negativamente y los clasifican así:

\section{Factores Externos}

* Evolución de la economía del país donde se ubica la empresa Historia de la empresa

* Evolución de la economía de los países donde la empresa trabaja Entorno en el que opera

* Evolución de la economía del sector al que pertenece la empresa

\section{Factores Internos}

* Factores comerciales (ventas, evolución de las mismas, clientes, competencia, etc.)

* Factores Técnicos (Capacidad productiva, tipo de tecnologías patentes etc.)

* Factores humanos (Tipo de dirección, formación de los trabajadores, motivación etc)

* Factores Financieros (Equilibrio, tesorería, beneficios, cash-flow etc)

* Otros factores: Juridicos, Administrativos etc 


\section{UNA MIRADA A LA VALORACIÓN EMPRESARIAL COMO MÉTODO AUTORITARIO EN LAS MIPYMES}

El mismo autor refleja que Las Normas Internacionales de Valoración presenta tres tipos de enfoque en función de la información sobre la que se basan:

\section{Enfoque basado en los activos \\ Enfoque de mercado \\ Enfoque de capitalización de rentas}

Esta clasificación, se puede ampliar en dos grupos más:

Métodos Mixtos o Métodos basados en el fondo de comercio o goodwill

Métodos basados en modelos multicriterio.

\section{Enfoque basado en los activos.}

Más conocidos como Métodos basados en el balance. Calculan el valor de la empresa partiendo de la información del balance y de la cuenta de resultados.

\section{Enfoque de Mercado. Valoración por múltiplos.}

Los métodos que comprende este grupo son comúnmente conocidos como Valoración por múltiplos y dentro de la terminología clásica de valoración quedarían comprendidos dentro del grupo de métodos comparativos.

Este grupo de métodos calcula el valor de una empresa comparándola con otras parecidas a ella mediante la utilización de unos ratios definidos a partir de la información de esas empresas comparables. En dichos Ratios siempre el numerador es el valor de las empresas comparables y el denominador un parámetro determinado (Beneficio, Ventas, Cashflow, etc.).

\section{Métodos mixtos o métodos basados en los divi- dendos y en el Fondo de comercio o Goodwill.}

Calculan el valor de una empresa partiendo de su Fondo de Comercio o Goodwill o bien de los dividendos.
Este método es comúnmente conocido como método por Descuento de Flujos de Caja o cash-flow (Discount cash-flow, DCF), y es el más utilizado y el que mayor crédito tiene dentro del ámbito de la valoración de este tipo de activos. Las NIV lo describen como "Estima el valor de una empresa, una participación empresarial o un título calculando el valor actual de los beneficios esperados"

\section{Métodos basados en modelos multicriterio.}

Estas técnicas están basadas en metodología que ayuda a la toma de decisiones en la empresa. Son métodos novedosos en valoración de activos y abren un campo de gran interés por la posibilidad de manejar e introducir en la valoración factores cualitativos e intangibles, así como su aplicación utilizando distintos expertos.

Los métodos vistos son fiables, sin embargo un factor clave para determinar el método de valoración para una Pyme debe ser decidir qué tan avanzado es su modelo de negocio. Si la Mipyme ha alcanzado cierto nivel de madurez y sus ingresos tienen posibilidad de ser vistos en el futuro con cierto nivel de confianza, un modelo estándar de flujo de efectivo descontado o equivalente puede ser una forma correcta de valoración. Si la Mipyme aún está creciendo y sigue una curva hacia la maduración, entonces el panorama competitivo juega un papel importante en la decisión de los multiplicadores de ingresos y ganancias para aplicar.

Se podría pensar que en materia de valoración y siendo un tanto impreciso "todo depende" en gran medida de la etapa del ciclo de vida de la empresa, la entidad que realiza la valoración y el objetivo concreto que se persigue. 


\section{UNA MIRADA A LA VALORACIÓN EMPRESARIAL COMO MÉTODO AUTORITARIO EN LAS MIPYMES}

\section{CONCLUSIONES}

uienes toman las decisiones rara vez podrán elegir basados en un solo criterio. Las diferentes teorías del valor demandan la evolución de sus formas de interpretación que den paso a un mayor acercamiento de lo que es aceptado en su momento. Las formas de valoración han ido evolucionando porque llegan como ideas que transforman la manera en que sentimos a la organización

Alrededor del valor hay intereses. Esta frase podría confundirse con la dinámica financiera del valor del dinero en el tiempo, pero aquí toma un matiz cuando los mecanismos de medición y valoración intentan formar una idea de qué es la riqueza que una empresa posee y cuál es la posición frente a un mercado incesante al que hay que explicarle qué es el valor y qué información se toma para generar una concepción alrededor de éste. Otro matiz severo de la realidad de las Mipymes, está asociada a la toma de decisiones y modulación de imagen, por supuesto bajo la austera perfección del financista que con maestría en los diferentes métodos provoca las expectativas de los stakeholders.

Los métodos de valoración en las empresas y específicamente en las Mipymes son transformables, y si lo son, no se está diciendo que los anteriores métodos hayan sido equivocados, sino que fueron el pie de fuerza que explicaba una realidad compleja pero que, al ser cambiante por estar sujeta a transformaciones socialmente aceptadas, se amolda para brindar una especie de necesaria seguridad y supuesta precisión aceptada por una forma de medición por autoridad.

Si el valor se basa en estimaciones y juicios en lugar de verdades absolutas, nos encontramos entonces con que son mediciones y valoraciones soportadas en el consenso social y no se puede desconocer que se dibujan sobre supuestos y modelos que llevan a pensar que el valor es una construcción social legitimada y considerada objetiva, pero edificada por la intersubjetividad. 


\section{UNA MIRADA A LA VALORACIÓN EMPRESARIAL COMO MÉTODO AUTORITARIO EN LAS MIPYMES}

\section{BIBLIOGRAFÍA}

Aristóteles (2000), Política, versión de Manuela García Valdés, Madrid: Gredos.

Aznar Bellver, J., Cayo Araya, T., \& Cevallos Varela, D. (2016). Valoración de empresas, métodos y casos para pequeñas y medianas empresas. Valencia: Editorial Ardiles.

Baas T. e M. Schroorten. (2006). Relationship Banking and SMEs: Theoretical Analysis. Small Business Economics , 127-137.

Bachelard,G. (1972): La Formación del Espíritu Científico. Buenos Aires: Siglo XXI Editores.

Behr, P. (2013). Financial constrataints of private firms and bank lending behavior. Journal of banking \& finance, 3472-3485.

Cruz Soto, L. A. (2010). El concepto de autoridad en el pensamiento de Aristóteles y su relación con el concepto de autoridad en el comportamiento administrativo. Obtenido de Contaduría y administración: http://www.scielo.org.mx/scielo.php? script $=$ sci_arttext\&pid=S0186-

10422010000200004

Damodaran, A. (2005). Valuation approaches and metrics: a survey of the theory and evidence. Delft: now Publishers Inc.

Fabelo Corzo, J. R. (2004). Los valores y sus desafíos actuales. Obtenido de https:// www.insumisos.com/lecturasinsumisas/Los $\%$ 20valores $\% 20 \mathrm{y} \% 2010 \mathrm{o} \% 20$ desafios $\%$

20actuales.pdf

Gómez Villegas, M. (2016). Medición y valoración contable, una aproximación heterodoxa. 3er Simposio Internacional de Contametría. Universidad Libre.

Fernández, P. (2013). Company valuation methods. IESE Business School.
Gadamer, Hans-Georg (2000). Verdad y Método, cuarta edición, Tr. Manuel Olasagasti, Salamanca.

Henric Coll, M. (2009). Las Falacias del Tecnomanagement. Autoedición.

Millanao M. , R., \& Saavedra R. , O. (2011). Método de valoración de empresas Pyme. Obtenido de Universidad de Chile: http://repositorio.uchile.cl/ handle/2250/113587

Morales de Barbenza, C. (2001). Consideraciones acerca de la objetividad en evaluacion psicologica. Obtenido de Interdisciplinaria: http:// www.redalyc.org/articulo.oa?id=18011325004

Pérez-Cotapos , G., \& Silva Palaveci, B. (2009). Valorización de empresas: La aplicación de una metodología. Valparaíso: Ediciones universitarias.

Revista Dinero. (2016). Mipymes generan alrededor del 67\% del empleo en Colombia. Dinero, 1-2.

Saavedra, M., \& Hernandez, Y. (2008). Aplicación de las Técnicas Metodólogicas y su relación con las características de las empresas y empresarios. Hidalgo.

Sánchez Hernández, A. (2005). Análisis filosófico del concepto valor. Humanidades Médicas, 1-23.

Torgerson, W.S.(1967): Theory and Methods of Scaling. Nueva York: John Wiley and Sons, Inc.

Uccelatore, M. (2012). importancia y financiamiento de las pequeñas y medianas empresas. Cecreda.

UNAM. (2017). Medición y recolección de datos. Obtenido de http://www.psicol.unam.mx/ Investigacion2/pdf/METO6F.pdf

Universidad EAFIT. (2017). ¿Qué son las finanzas? Obtenido de http://www.eafit.edu.co/escuelas/ economiayfinanzas/departamento-finanzas/acercadel-departamento/Paginas/que-son-lasfinanzas.aspx

Van Praag C. M. e P. H. Versloot. (2007). What is the value of entrepreneurship? A review of recent 


\section{UNA MIRADA A LA VALORACIÓN EMPRESARIAL COMO MÉTODO AUTORITARIO EN LAS MIPYMES}

finanzas.aspx

Van Praag C. M. e P. H. Versloot. (2007). What is the value of entrepreneurship? A review of recent research. Small Business Economics, 351-382. 